\title{
Decoherence of quantum discord in an asymmetric-anisotropy spin system
}

\author{
Xiang Hao.* Chunlan Ma, and Jinqiao Sha \\ Department of Physics, School of Mathematics and Physics, \\ Suzhou University of Science and Technology, \\ Suzhou, Jiangsu 215011, People's Republic of China
}

\begin{abstract}
The decoherence of quantum correlation is investigated in the Heisenberg spin system with the asymmetric anisotropic interactions. The quantum entanglement and discord are used to quantify the quantumness of the correlations. By the analytical and numerical methods, we find that quantum discord decays asymptotically in time under the effects of the independent local Markovian reservoirs. This is markedly different from the sudden change of the entanglement. Before the disappearance of the entanglement, the dynamic behaviour of quantum discord is very similar to that of the entanglement. It is also shown that the discord declines rapidly for the interacting spin system compared with the case of noninteracting qubits. At an arbitrary finite temperature, the nonzero thermal discord can be enhanced by the asymmetric anisotropic interactions which induce quantum fluctuations.
\end{abstract}

PACS: 03.65.Ta, 03.67.Mn, 75.10.Jm, 75.10.Pq

Keywords: Decoherence, Quantum discord, Asymmetric anisotropic interaction

\footnotetext{
${ }^{*}$ Corresponding author,Email:110523007@suda.edu.cn
} 


\section{INTRODUCTION}

The quantum aspects of correlations in composite systems are a key issue in quantum information theory [1]. Quantum entanglement is extensively regarded as one useful measure of nonlocal coherence. The inseparable (entangled) states are applicable in many parts of quantum information processing, like quantum teleportation, cryptography, dense coding and quantum computation [2-4]. However, the recent theoretical and experimental developments have discovered that some separable (nonentangled) mixed states are useful for improving performance in some tasks of quantum computers [5-7]. The entanglement is not the only type of quantum correlation. To generally quantify the quantumness of correlations contained in bipartite systems, Ollivier and Zurek have defined a measure known as quantum discord [8]. It is largely accepted that the total correlations contained in quantum states can be described by quantum mutual information which is a sum of the quantum discord and classical correlation given by Henderson and Vedral [9, 10]. Quantum discord has received much attention in theoretical studies [11 17]. By means of the thermodynamics

of correlations, quantum discord can exhibit signatures of quantum phase transitions [12]. As is well known, the composite system loses unavoidably the quantum coherence due to interacting with the environments[18, 19]. The dynamics of the discord were also analyzed in the system of noninteracting qubits coupled to the environments [14]. The robustness of quantum discord is beneficial for the realization of scalable quantum computing in contrast to the fragility of the entanglement [20].

Because of the fundamental and practical values, it is necessary to investigate the dynamic properties of quantum discord in some concrete systems of interacting spins. In semiconductor quantum dots and single molecular magnets, the magnetic properties of these systems were usually studied by the Heisenberg spin models with the asymmetric anisotropic couplings such as Dzyaloshinskii-Moriya interactions [21 25]. In this paper, the Markovian decoherence of the quantum discord is obtained by the master equation when the independent thermal reservoirs are considered. In section II, the time-dependent behaviour of quantum discord and entanglement are analytically and numerically analyzed. Compared with the case of noninteracting spin systems, the effects of spin interactions on the decoherence of quantum discord are taken into account. In section III, the thermal property of the quantum discord is also investigated. Finally, a simple discussion concludes the paper. 


\section{THE MARKOVIAN DYNAMICS OF QUANTUM CORRELATION}

To clearly describe the dynamics of quantum correlation, we use two types of measures including the entanglement of formation and quantum discord. For an arbitrary bipartite state $\rho_{A B}$, the total correlations are expressed by quantum mutual information [26]

$$
I\left(\rho_{A B}\right)=\sum_{i=A, B} S\left(\rho_{i}\right)-S\left(\rho_{A B}\right),
$$

where $\rho_{i}$ represents the reduced density matrix of subsystem $i$ and $S(\rho)=-\operatorname{Tr}\left(\rho \log _{2} \rho\right)$ is the von Neumann entropy. Henderson and Vedral proposed one measure of bipartite classical correlation $C\left(\rho_{A B}\right)$ based on a complete set of local projectors $\left\{\Pi_{B}^{k}\right\}$ on one subsystem $B$. After the local measurements, the reduced state of subsystem $A$ can be written by

$$
\rho_{A}^{k}=\frac{1}{p^{k}} \operatorname{Tr}_{B}\left[\left(\mathbf{1}_{A} \otimes \Pi_{B}^{k}\right) \rho_{A B}\left(\mathbf{1}_{A} \otimes \Pi_{B}^{k}\right)\right]
$$

Here $p^{k}$ is the measurement probability for the $k$-th local projector and $\mathbf{1}_{A}$ is the identity operator on subsytem $A$. When subsystem $B$ is described in the two-dimensional Hilbert space $\{|0\rangle,|1\rangle\}$, a complete set of the local measurements can be given by $\left\{\Pi_{B}^{k}=V|k\rangle\langle k| V^{\dagger},(k=\right.$ $0,1)\}$ where the arbitrary unitary operation $V(\theta, \phi)$ for $\theta \in[0, \pi]$ and $\phi \in[0,2 \pi]$ is

$$
V(\theta, \phi)=\left(\begin{array}{cc}
\cos \frac{\theta}{2} & \sin \frac{\theta}{2} e^{-i \phi} \\
\sin \frac{\theta}{2} e^{i \phi} & -\cos \frac{\theta}{2}
\end{array}\right) .
$$

Then the classical correlation in the bipartite quantum state $\rho_{A B}$ can be given by

$$
C\left(\rho_{A B}\right)=S\left(\rho_{A}\right)-\sup _{\left\{\Pi_{B}^{k}\right\}}\left\{S\left(\rho_{A \mid B}\right)\right\}
$$

where $S\left(\rho_{A \mid B}\right)=\sum_{k=0,1} p^{k} S\left(\rho_{A}^{k}\right)$ is the conditional entropy of subsystem $A$ and $\sup \left\{S\left(\rho_{A \mid B}\right)\right\}$ signifies the minimal value of the entropy with respect to a complete set of local measurements $\left\{\Pi_{B}^{k}\right\}$. Quantum discord is simply defined by

$$
Q\left(\rho_{A B}\right)=I\left(\rho_{A B}\right)-C\left(\rho_{A B}\right)
$$

In the following study, we draw on the master equation to analyze the time-dependent behaviour of quantum discord in the quantum system with the asymmetric anisotropic interaction. For a two-qubit system, the total Hamiltonian is written by $H=H_{0}+H_{I}$ where the intrinsic Hamiltonian of two qubits $H_{0}=\frac{\omega}{2}\left(\sigma_{A}^{z}+\sigma_{B}^{z}\right)$ and the interacting Hamiltonian 
$H_{I}=\frac{J}{2}\left[\sum_{\alpha=x, y, z} \sigma_{A}^{\alpha} \sigma_{B}^{\alpha}+\vec{D} \cdot\left(\vec{\sigma}_{A} \times \vec{\sigma}_{B}\right)\right]$. To lay stress on the effects of the interacting Hamiltonian on quantum discord, we assume that $H_{0} \ll H_{I}$. Here $\sigma^{\alpha}$ is the pauli operator. For the case of antiferromagnetic couplings $J>0$, the asymmetric anisotropy $\vec{D}=D \vec{e}_{z}$ is considered. It is reasonable to assume that two qubits are coupled to its local thermal reservoirs $E_{A}, E_{B}[20]$. Suppose that the initial state at $t=0$ is $\rho(0)=\rho_{A B}(0) \otimes\left(\left|0_{E_{A}} 0_{E_{B}}\right\rangle\left\langle 0_{E_{A}} 0_{E_{B}}\right|\right)$ where $\left|0_{E_{A}} 0_{E_{B}}\right\rangle$ denotes the vacuum state of the two local reservoirs. The evolution of quantum state between qubit $A$ and $B$ is given by the master equation

$$
\dot{\rho}_{A B}(t)=-i\left[H, \rho_{A B}\right]+\hat{L}\left(\rho_{A B}\right),
$$

where the Lindbald operator

$$
\begin{array}{r}
\hat{L}(\rho)=\sum_{i=A, B}\left(\bar{n}_{i}+1\right) \gamma_{i}\left(2 \sigma_{i}^{-} \rho \sigma_{i}^{+}-\rho \sigma_{i}^{+} \sigma_{i}^{-}-\sigma_{i}^{+} \sigma_{i}^{-} \rho\right) \\
+\bar{n}_{i} \gamma_{i}\left(2 \sigma_{i}^{+} \rho \sigma_{i}^{-}-\rho \sigma_{i}^{-} \sigma_{i}^{+}-\sigma_{i}^{-} \sigma_{i}^{+} \rho\right) .
\end{array}
$$

Here $\bar{n}_{i}=\bar{n}$ is the mean number of the thermal reservoir and $\gamma_{i}=\gamma$ signifies the rate of spontaneous emission for each qubit. In fact, the temperatures of the thermal reservoirs can be varied from zero values to small ones after the local operations. At very low temperatures, the initial state is approximately the ground state $\rho(0)$. The mean number $\bar{n}=\frac{1}{\exp \left(\omega_{E} / k_{B} T\right)-1}$ could be a nonzero value where $\omega_{E}$.

We adopt the numerical approach to calculate the density matrix at any time. For numerical purposes, it is convenient to regard the components of density matrix $\rho$ as being written as a single column vector $\tilde{\rho}$ in Matlab. Then the pre-multiplication of the operator $\sigma \rho$ can be written as $\operatorname{spre}(\sigma) \tilde{\rho}$ where the function $\operatorname{spre}(\sigma)$ can be given by the Kronecker product of the identity and matrix $\sigma$. In the same way, the post-multiplication of the operator $\rho \sigma$ can also be written as spost $(\sigma) \tilde{\rho}$. Therefore, the master equation in Eq. (6) can also be expressed by $\dot{\tilde{\rho}}_{A B}(t)=\mathcal{L} \tilde{\rho}_{A B}(0)$ where the superoperator $\mathcal{L}$ is obtained by the pre-multiplication and post-multiplication of the operators. Because the superoperator $\mathcal{L}$ is time-independent and is small enough to be diagonalized numerically, the $i-t h$ component of $\tilde{\rho}(t)$ can be calculated by $\rho_{i}(t)=\sum_{j} a_{i j} \exp s_{j} t$. Here $s_{j}$ is the eigenvalue of $\mathcal{L}$ and satisfies $\mathcal{L}_{i k}=\sum_{j} U_{i j} s_{j} U_{j k}^{-1}$. The coefficients $a_{i j}=U_{i j} \sum_{k} U_{j k}^{-1} \tilde{\rho}_{k}(0)$. The numerical calculation of $a_{i j}$ and $s_{j}$ is completed by the program of exact diagonalization of the superoperator $\mathcal{L}$.

From the point of view of the practice, the initial state $\rho_{A B}(0)$ between qubit $A$ and $B$ is 
usually chosen to be the ground state which is expanded in the product space of two qubits

$$
\left|\psi_{g}\right\rangle=\frac{1}{\sqrt{2}}\left(|01\rangle+e^{i \alpha}|10\rangle\right)
$$

where $e^{i \alpha}=\frac{1+i D}{\sqrt{1+D^{2}}}$. It is clearly seen that the ground state is just one maximally entangled Bell state. The general expression of quantum state $\rho_{A B}(t)$ satisfies

$$
\rho_{A B}(t)=\left(\begin{array}{cccc}
u & 0 & 0 & 0 \\
0 & x & z & 0 \\
0 & z^{*} & y & 0 \\
0 & 0 & 0 & v
\end{array}\right) .
$$

According to the result of [16], the analytical solution of quantum correlation can be given by

$$
Q\left(\rho_{A B}\right)=S\left(\rho_{B}\right)-S\left(\rho_{A B}\right)+\min \left\{S_{0}, S_{1}\right\} .
$$

The entropy is $S_{0}=-\left(u \log _{2} \frac{u}{u+y}+y \log _{2} \frac{y}{u+y}+x \log _{2} \frac{x}{x+v}+v \log _{2} \frac{v}{x+v}\right)$ and $S_{1}=$ $-\sum_{q=0,1} \frac{1+(-1)^{q} \theta_{1}}{2} \log _{2} \frac{1+(-1)^{q} \theta_{1}}{2}$ where $\theta_{1}=\sqrt{(u+x-y-v)^{2}+4|z|^{2}}$. Meanwhile, the entanglement of formation can be used to evaluate the quantum correlation, According to [27 29], the value of the entanglement is obtained by

$$
E\left(\rho_{A B}\right)=2 \max \{|z|-\sqrt{u v}, 0\} .
$$

Through the numerical approach, the decoherence of quantum correlation can be given by Fig. 1(a) and 1(b). If qubits $A$ and $B$ are in the ground state, the maximally entangled ground state has the maximal value of quantum correlation, $E\left(\rho_{A B}\right)=Q\left(\rho_{A B}\right)=1$ at the time $t=0$. In Fig. 1(a), it is clearly shown that the values of quantum discord $Q\left(\rho_{A B}\right)$ are decreased and infinitely close to zero with time. The nonvanishing phenomenon of quantum discord is different from the sudden disappearance of the entanglement. In the vicinity of $E\left(\rho_{A B}\right)=0$, the nonzero values of the discord can also quantify the nonclassical correlation for separable mixed states which make possible some tasks of quantum computers. However, it is noted that the decay of quantum discord is very similar to that of the entanglement before the time of the vanishing of entanglement. This aspect demonstrates that quantum discord can describe the nonlocal coherence like the entanglement. It is also valuable to investigate the case where qubits are initially in a separable state $\psi_{A B}(0)=|10\rangle$. From Fig. 1(b), we find that quantum discord and entanglement can be generated and then vanish in time. The behaviour arises from the coupling between qubit $A$ and $B$. 
For a special case of $J=0$, the evolution of quantum state $\rho_{A B}(t)$ can be described by a completely positive trace-preserving map [30]. For a general two-qubit state $\rho_{A B}(0)=\sum_{k l, m n} a_{m n, k l}|k\rangle_{A}\langle m|\otimes| l\rangle_{B}\langle n|$, the evolved state in time can be written by $\rho_{A B}(t)=\sum_{k l, m n} \sum_{j=0}^{3} \sum_{j^{\prime}=0}^{3} a_{m n, k l}\left(K_{A j}|k\rangle_{A}\langle m| K_{A j}^{\dagger}\right) \otimes\left(K_{B j^{\prime}}|l\rangle_{B}\langle n| K_{B j^{\prime}}^{\dagger}\right)$ where the Kraus operators $K_{i 0}=\sqrt{\frac{\bar{n}+1}{2 \bar{n}+1}}\left(|0\rangle_{i}\langle 0|+\sqrt{1-p}| 1\rangle_{i}\langle 1|\right), K_{i 1}=\sqrt{\frac{(\bar{n}+1) p}{2 \bar{n}+1}}|0\rangle_{i}\langle 1|, \quad K_{i 2}=$ $\sqrt{\frac{\bar{n}}{2 \bar{n}+1}}\left(\sqrt{1-p}|0\rangle_{i}\langle 0|+| 1\rangle_{i}\langle 1|\right)$ and $K_{i 3}=\sqrt{\frac{\bar{n} p}{2 \bar{n}+1}}|1\rangle_{i}\langle 0|$. Here $|0(1)\rangle_{i}$ is the ground(excited) state of qubits $i=A, B$ satisfying $\sigma_{i}^{z}|0(1)\rangle_{i}=\mp|0(1)\rangle_{i}$ and $p(t)=1-e^{\frac{\gamma(2 \bar{n}+1) t}{2}}$ means the probability of the atom exchanging a quantum with the reservoir. If the initial state is $|\psi\rangle_{g}$, the expression of $\rho_{A B}(t)$ is similar to Eq. (9),

$$
\begin{aligned}
& u=\frac{\bar{n} p}{2 \bar{n}+1}\left[1-\frac{(\bar{n}+1) p}{2 \bar{n}+1}\right], v=\frac{(\bar{n}+1) p}{2 \bar{n}+1}\left(1-\frac{\bar{n} p}{2 \bar{n}+1}\right), \\
& x=\frac{1}{2}\left[\frac{\bar{n}(\bar{n}+1) p^{2}}{(2 \bar{n}+1)^{2}}+\left(1-\frac{\bar{n} p}{2 \bar{n}+1}\right)\left(1-\frac{(\bar{n}+1) p}{2 \bar{n}+1}\right)\right], \\
& z=\frac{1}{2}(1-p), y=1-u-x-v .
\end{aligned}
$$

Therefore, the angle $\theta_{1}$ of the entropy $S_{1}$ in Eq. (10) is analytically expressed by $\theta_{1}=$ $\sqrt{\frac{p^{2}}{(2 \bar{n}+1)^{2}}+(1-p)^{2}}$. From the above analysis, it is seen that quantum discord is independent on the asymmetric anisotropy $D$ if the initial state is the ground state $\left|\psi_{g}\right\rangle$. In Fig. 2, two cases of the decoherence of quantum discord are shown when the interactions between two qubits are $J=0$ and $J=1$ respectively. It is found that the values of quantum discord rapidly decline to very small ones under the impact of the interaction $J \neq 0$. This manifests that the dramatic loss of nonclassical correlation happens when there is the strong interaction between qubits in the system.

\section{THERMAL DECOHERENCE OF QUANTUM DISCORD}

In general, states of qubits for quantum information processing are usually influenced by thermal temperatures. Under the effect of the temperatures, the thermal decoherence is unavoidable. Therefore, it is of value to study the properties of quantum discord for the thermal equilibrium state. In the spin model, the mixed state $\rho(T)$ at any equilibrium temperature $T$ is expressed by

$$
\rho(T)=\frac{1}{Z} \sum_{j}\left|\psi_{j}\right\rangle\left\langle\psi_{j}\right| \exp \left(-\epsilon_{j} / k T\right),
$$


where the partition function $Z=\sum_{j} \exp \left(-\epsilon_{j} / k T\right)$ and $\left|\psi_{j}\right\rangle, \epsilon_{j}$ are the $j-t h$ eigenstate of the Hamiltonian $H$ and corresponding eigenvalue respectively. The exact diagonal expression of $H$ is given by a set of eigenstates and corresponding eigenvalues,

$$
\begin{aligned}
\epsilon_{1,2} & =J\left(-\frac{1}{2} \pm|\eta|\right),\left|\psi_{1,2}\right\rangle=\frac{1}{\sqrt{2}}\left(|01\rangle \pm \frac{\eta}{|\eta|}|10\rangle\right) ; \\
\epsilon_{3} & =\frac{J}{2}-\omega,\left|\psi_{3}\right\rangle=|00\rangle ; \epsilon_{4}=\frac{J}{2}+\omega,\left|\psi_{4}\right\rangle=|11\rangle .
\end{aligned}
$$

Here the parameter $\eta=1+i D$. Because the thermal state has the same form in Eq. (9), the calculation of quantum discord is also solved by Eq. (10). The thermal properties of quantum discord are shown in Fig. 3. It is found that the asymmetric anisotropy $D$ can increase the values of quantum discord at any temperature $T$. This is the reason that the asymmetric anisotropy along the $z$ direction induces the quantum fluctuation in the $X Y$ plane which can enhance the quantum correlation. The decay of quantum discord occurs when the temperatures $T$ are increased. For finite temperatures, the values of quantum discord are always nonzero. The result coincides with that of [31]. As is well known, the thermal entanglement is gradually decreased to zero with the increase of the temperatures

[28]. In the context, it is the fact that the robustness of quantum discord against the decoherence is helpful for the realization of quantum computing.

\section{DISCUSSION}

Using quantum discord, we mainly investigate the decoherence of quantum correlation for two interacting qubits coupled to independent reservoirs. Compared with the case of noninteracting qubits, quantum correlation of the states in the interacting qubits system decays very rapidly. The values of quantum discord decrease asymptotically in time. This nonvanishing phenomenon is apparently different from the sudden change of the entanglement. It is found that the evolution behaviour of quantum discord is very similar to that of the entanglement before the sudden disappearance of the entanglement. This demonstrates that quantum discord for inseparable states can describe the nonlocal coherence like the entanglement. Moreover, after the sudden death of the entanglement, the nonzero values of quantum discord also manifest the existence of quantum correlation for separable mixed states. The thermal decoherence from the temperature is also considered. It is shown that the nonvanishing quantum discord at any finite temperature can be increased by the asym- 
metric anisotropy. Quantum discord also declines asymptotically with the temperature. Therefore, quantum discord is a general quantifier for nonclassical correlation.

\section{ACKNOWLEDGEMENTS}

The work was supported by the Research Program of Natural Science for Colleges and Universities in Jiangsu Province Grant No. 09KJB140009 and the National Natural Science Foundation Grant No. 10904104.

[1] M. A. Nielsen and I. L. Chuang, Quantum Computation and Quantum Information(Cambridge University Press, Cambridge, 2000).

[2] C. H. Bennett, G. Brassard, C. Crépeau, R. Jozsa, A. Peres, and W. K. Wootters, Phys. Rev. Lett. 70 (1993) 1895.

[3] D. Loss and D. P. DiVincenzo, Phys. Rev. A 57 (1998) 120.

[4] R. Horodecki, P. Horodecki, M.Horodecki, and K. Horodecki, Rev. Mod. Phys. 81 (2009) 865.

[5] D. A. Meyer, Phys. Rev. Lett. 85 (2000) 2014.

[6] A. Datta, A. Shaji, and C. M. Caves, Phys. Rev. Lett. 100 (2008) 050502.

[7] B. P. Lanyon, M. Barbieri, M. P. Almeida, and A. G. White, Phys. Rev. Lett. 100 (2008) 200501.

[8] H. Ollivier and W. H. Zurek, Phys. Rev. Lett. 88 (2001) 017901.

[9] L. Henderson and V. Vedral, J. Phys. A 34 (2001) 6899.

[10] V. Vedral, Phys. Rev. Lett. 90 (2003) 050401.

[11] S. Luo, Phys. Rev. A 77 (2008) 022301.

[12] M. S. Sarandy, Phys. Rev. A 80 (2009) 022108.

[13] R. Dillenschneider and E. Lutz, Europhys. Lett. 88 (2009) 50003.

[14] T. Werlang, S. Souza, F. F. Fanchini, and C. J. Villas-Boas, Phys. Rev. A 80 (2009) 024103.

[15] J. Cui and H. Fan, J. Phys. A 43 (2010) 045305.

[16] Mazhar Ali, A. R. P. Rau, and G. Alber, Phys. Rev. A 81 (2010) 042105.

[17] J. Maziero, T. Werlang, F. F. Fanchini, L. C. Céleri, and R. M. Serra, Phys. Rev. A 81 (2010) 022116. 
[18] C. A. R. Rosario, K. Modi, A. M. Kuah, A. Shaji, and E. C. G. Sudarshan, J. Phys. A: Math. Theor. 41 (2008) 205301.

[19] D. W. Leung, J. Math. Phys. 44 (2003) 528.

[20] T. Yu and J. H. Eberly, Phys. Rev. Lett. 93 (2004) 140404.

[21] D. C. Dender, P. R. Hammar, D. H. Reich, C. Broholm, and G. Aeppli, Phys. Rev. Lett.79 (1997) 1750.

[22] H. De Raedt, S. Miyashita, K. Michielsen, and M. Machida, Phys. Rev. B 70 (2004) 064401.

[23] I. Dzyaloshinskii, Phys. Chem. Solids 4 (1958) 241.

[24] T. Moriya, Phys. Rev 120 (1960) 91.

[25] L. S. Levitov and E. I. Rashba, Phys. Rev. B 67 (2001) 115324.

[26] B. Groisman, S. Popescu, and A. Winter, Phys. Rev. A 72 (2005) 032317.

[27] X. Wang and P. Zanardi, Phys. Lett. A301 (2002) 1.

[28] M. C. Arnesen, S. Bose and V. Vedral, Phys. Rev. Lett. 87 (2001) 017901.

[29] A. Bayat and V. Karimipour, Phys. Rev. A71 (2005) 042330.

[30] L. Aolita, R. Chaves, D. Cavalcanti, A. Acín, and L. Davidovich, Phys. Rev. Lett. 100 (2008) 080501 .

[31] T. Werlang and G. Rigolin, Phys. Rev. A 81 (2010) 044101. 


\section{Figure caption}

Figure 1

As two different quantifiers of quantum correlation, the quantum discord (solid line) and entanglement (dashed line) are numerically calculated and plotted as a function of the decoherence time. The mean number of the thermal reservoir is chosen to be $\bar{n}=1$ and the rate of spontaneous emission is $\gamma=0.1$. The parameters are chosen to be $J=1, D=0.2$ and $\omega=0.1$. (a). The qubits are initially in the ground state $\left|\psi_{A B}(0)\right\rangle=\left|\psi_{g}\right\rangle$; (b). The initial state of the qubits is a separable one $\left|\psi_{A B}(0)\right\rangle=|10\rangle$.

Figure 2

The decoherence of quantum discord for the two-qubit system is illustrated under the independent reservoirs. The solid line denotes the case of two interacting qubits in the condition of $J=1, D=0.2$ and $\omega=0.1$. The dashed line signifies the other case of two noninteracting qubits for $J=0$. The mean number of the thermal reservoir is chosen to be $\bar{n}=1$ and the rate of spontaneous emission is $\gamma=0.1$.

Figure 3

The quantum discord at the thermal equilibrium state for $J=1$ and $\omega=0.1$ is shown with the changes of the temperature $T$ and asymmetric anisotropic interaction $D$. 

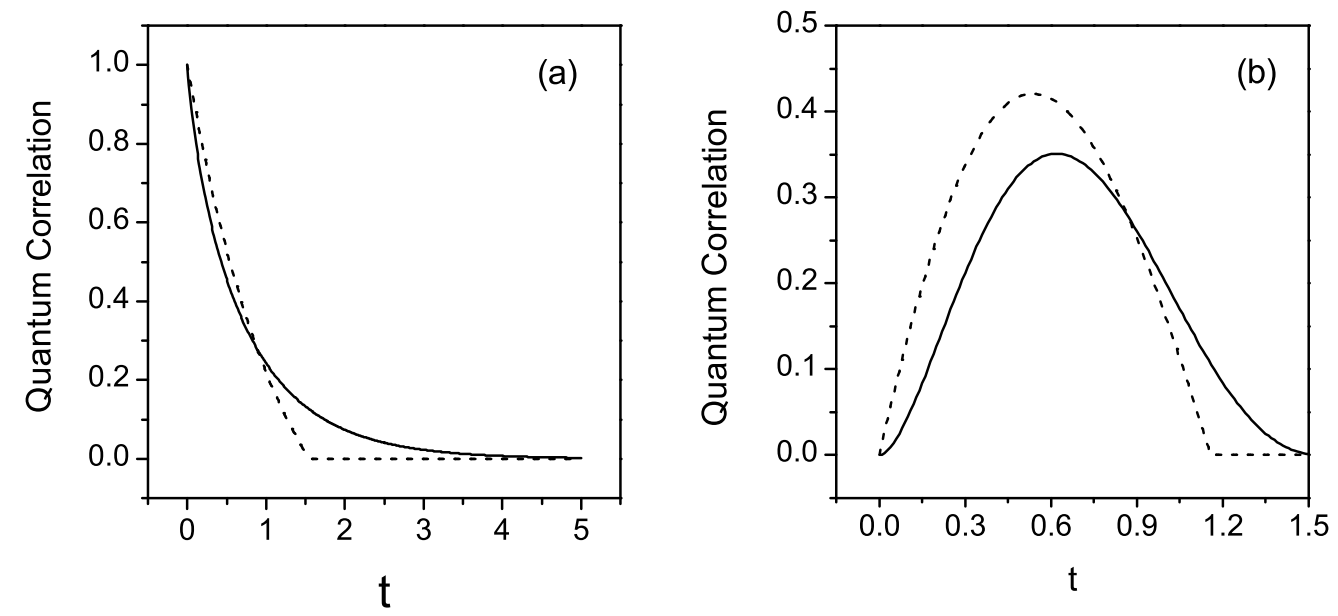

Fig. 1 


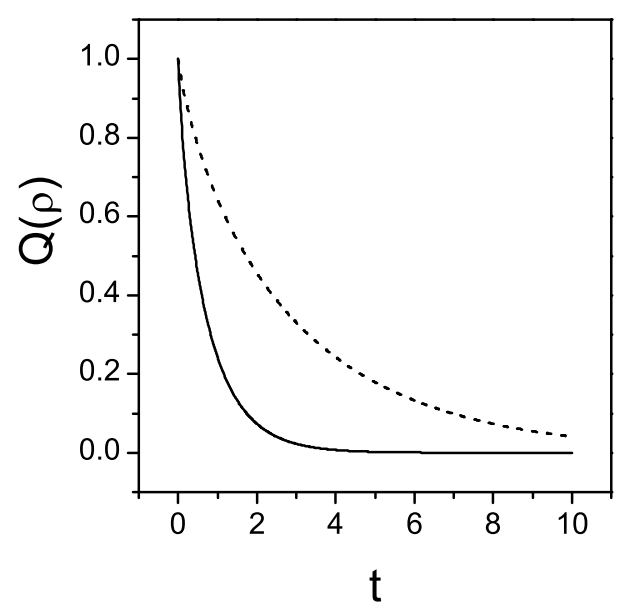

Fig. 2 


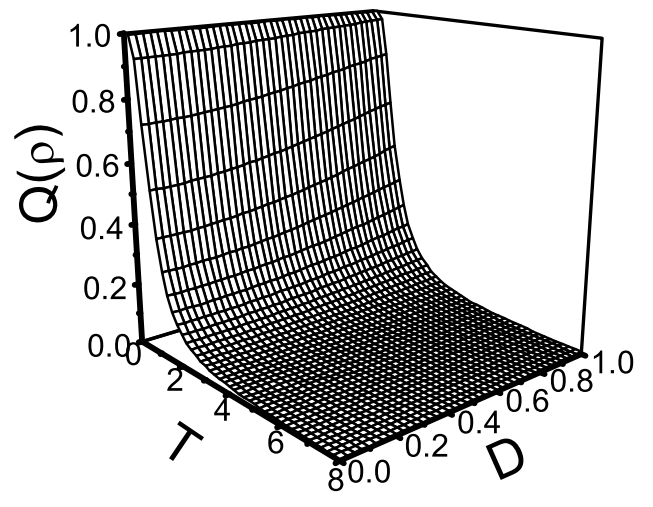

Fig. 3 